IdeAs

Idées d'Amériques

$11 \mid 2018$

Modernités dans les Amériques : des avant-gardes à aujourd'hui

\title{
1968 aux Etats-Unis, tournant politique des années de mouvement
}

James Cohen

\section{(2) OpenEdition \\ Journals}

Édition électronique

URL : https://journals.openedition.org/ideas/2742

DOI : $10.4000 /$ ideas. 2742

ISSN : 1950-5701

Éditeur

Institut des Amériques

Référence électronique

James Cohen, "1968 aux Etats-Unis, tournant politique des années de mouvement », IdeAs [En ligne],

11 | 2018, mis en ligne le 18 juin 2018, consulté le 18 octobre 2022. URL : http://

journals.openedition.org/ideas/2742 ; DOI : https://doi.org/10.4000/ideas.2742

Ce document a été généré automatiquement le 18 octobre 2022.

\section{(c) (1) (9)}

Creative Commons - Attribution - Pas d'Utilisation Commerciale - Pas de Modification 4.0 International - CC BY-NC-ND 4.0

https://creativecommons.org/licenses/by-nc-nd/4.0/ 


\title{
1968 aux Etats-Unis, tournant politique des années de mouvement
}

\author{
James Cohen
}

1 James Cohen est professeur à l'Université de Paris 3 Sorbonne Nouvelle, CREW (EA 4399), auteur de À la poursuite des «illégaux». Politiques et mouvements anti-immigrés aux États-Unis, Éditions Le Croquant, 2012.

2 Aux États-Unis, l'année 1968 se situe au milieu d'une grande vague de mouvements contestataires qui prend son essor au début des années 60 et se poursuit jusqu'au début des années 1970. Saisie de manière strictement événementielle, l'année est exceptionnellement riche en événements polarisants : affrontements de rue, actes de défiance des autorités civiles et militaires, assassinats d'acteurs de premier plan, enfin l'échéance électorale du mois de novembre dont les ondes de choc polarisantes n'ont pas fini de se faire sentir. Comme conjoncture politique, l'année pose de manière centrale la question du rapport de forces politiques entre les diverses formes d'opposition politique radicale constituant «le mouvement» et les forces conservatrices qui s'y opposaient.

Le bilan de ce rapport de forces apparaît comme double et contradictoire : c'est à la fois le moment où plusieurs mouvements à vocation « radicale » ou révolutionnaire entrent dans leur phase d'expansion la plus rapide, et le moment où, pour les conservateurs, un début de reprise en main de l'autorité devient possible. Pour citer l'historien et acteur militant de la période, Max Elbaum, il n'a jamais été plus évident qu'en 1968 que, pour des dizaines de milliers de personnes "le système était devenu la cible ${ }^{1}$. C'est pourtant aussi le moment où la réaction conservatrice reprend l'initiative politique, notamment, mais pas seulement, par la victoire du Républicain Richard Nixon à l'élection présidentielle du 5 novembre.

4 Lorsqu'on situe l'analyse de l'année 68 dans une discussion plus large sur la signification de toute la période de la montée contestataire, puis de son déclin, plusieurs interprétations s'affrontent. Ici nous en retiendrons deux pour les confronter: premièrement, celle représentée par le livre de Todd Gitlin The Sixties: Years of Hope, Days of Rage (1987), qui construit l'histoire de la période autour du 
mouvement étudiant dont à l'époque l'auteur faisait partie en tant que dirigeant (1963-1964) de Students for a Democratic Society (SDS), organisation étudiante centrale jusqu'à son éclatement en 1968. Selon Gitlin, le mouvement dans son ensemble s'est autodétruit en 1968 et a sombré dans une incohérence irrécupérable. Sans négliger le versant populaire des mobilisations, notamment les luttes multiformes des minorités, il a tendance à placer le mouvement étudiant, à grande majorité blanche, et son avantgarde intellectuelle, au centre de l'histoire. L'éclatement du SDS a signifié pour Gitlin l'incapacité de ladite "Nouvelle Gauche » à se renouveler durablement à cause d'une forte tendance à l'auto-sabotage. L'élection de Nixon confirme de ce point de vue la fin d'une période où la transformation pouvait être imaginée.

5 Autre acteur de la période devenu historien, Max Elbaumne partage pas ce bilan qu'il estime trop limité dans son angle de vue et trop manichéen. Implicitement, Gitlin divise la période en "good sixties » et "bad sixties ", le mauvais ayant fini par miner et détruire le bon. Elbaum ne nie pas l'implosion de SDS ou l'aventurisme ultragauchiste de certaines de ses composantes. Cependant, selon Elbaum, le récit de Gitlin fait oublier ou passer au second plan de nombreux acteurs qui ne sont pas de moindre importance que le SDS et qui, pour certains, ont continué à jouer un rôle actif après 1968 : « La baisse de l'activité militante parmi les étudiants blancs après 1970 et le tournant clandestin accompagné d'attentats à la bombe entrepris par quelques-uns ne sont qu'un élément d'un récit plus ample $»^{2}$. Ancien dirigeant d'un courant pro-chinois, Elbaum se réfère en particulier à une constellation d'organisations d'inspiration marxiste-léniniste qui ont contribué, comme il le démontre, à un cycle de luttes ouvrières, antiracistes et antiimpérialistes au début des années 1970, à partir d'une base sociale militante, plus noire, latino et asiatique que partout ailleurs à gauche. Il admet toutes les limites de ces courants (tendances dogmatiques et dépendant des relations sino-soviétiques, tâtonnements dans l'analyse, etc.) mais leur reconnaît néanmoins une connaissance des terrains syndicaux et populaires et une capacité à y implanter une culture critique et à y forger un capital d'expérience militante.

6 Le débat ouvert par les livres de Gitlin et Elbaum est très loin d'épuiser la discussion historiographique, mais il la fait avancer autrement en démontrant, par deux lectures distinctes, que la manière de restituer cette histoire n'est pas seulement une affaire de spécialistes, elle a aussi de profondes implications politiques contemporaines.

\section{Le Vietnam au centre de la radicalisation}

7 Si nous nous focalisons principalement sur la scène états-unienne, il va de soi que la perspective doit également se transnationaliser, afin de rendre compte des mouvements de contestation - très différents mais comparables et en partie « connectés »- dans plusieurs pays (France, Allemagne, Royaume-Uni, Japon, Mexique, Tchécoslovaquie, etc.). La contestation de la guerre du Vietnam n'était pas le seul point commun entre ces mouvements mais elle a joué un rôle central ${ }^{3}$.

8 Fin janvier 1968, le Front de libération nationale (FLN) fait une démonstration de force inattendue en s'emparant brièvement de plusieurs villes du Sud Vietnam, dont Saigon, où il a réussi à attaquer la base aérienne, le palais présidentiel et l'ambassade des ÉtatsUnis. L'offensive du Têt produit une série de chocs politiques. Les élites qui élaboraient la stratégie de la guerre ne pouvaient plus se cacher derrière un langage technocratique pour prétendre qu'elle était en train d'être "gagnée »" Le Pentagone 
était discrédité et la responsabilité du président Lyndon Johnson engagée. Son premier mandat électif prenait fin début 1969 mais sa réélection s'annonçait difficile. Les premières élections primaires du printemps 1968 révèlent une forte poussée de candidats favorables au retrait des troupes. Le 12 mars, à l'élection primaire du New Hampshire, le sénateur Eugene McCarthy du Minnesota obtient $42 \%$ des voix contre $48 \%$ pour Johnson qui, le 31 mars, renonce à un deuxième mandat.

Le mouvement antiguerre a célébré comme une victoire le retrait de Johnson ${ }^{5}$. Ce mouvement, en 1968, était en plein essor après la manifestation de Washington du 21 octobre 1967, où participent 100000 personnes. Le massacre du village de My Lai sous les ordres du lieutenant William Calley, le 16 mars 1968, radicalise le rejet de la guerre dans l'opinion. Une grève nationale d'étudiants et de lycéens contre la guerre mobilise environ un million de personnes le 26 avril $1968^{6}$. Le mouvement antiguerre allait connaître des mobilisations beaucoup plus impressionnantes encore en 1969 et 1970, lorsque Nixon et Kissinger intensifient les bombardements en les étendant au Laos et au Cambodge.

\section{Des droits civiques au Black Power}

10 Les lois conquises par le mouvement des droits civiques (Civil Rights Act, 1964 et Voting Rights Act, 1965) ouvrent la voie à la reconnaissance de droits dans de nombreux domaines. En 1968 sont votées une loi sur l'accès équitable au logement (Fair Housing Act) et une loi permettant l'enseignement bilingue de transition pour des élèves de langue maternelle autre que l'anglais (Bilingual Education Act). Sur plan intérieur, l'administration Johnson est la plus à « gauche » de l'histoire. Les programmes sociaux de la Great Society représentent la première poussée de l'État-Providence aux États-Unis depuis les années 1930.

11 Cela ne signifiait nullement que les inégalités sociales à connotation ethno-raciale étaient en train de disparaître. À l'occasion de la publication du rapport Kerner sur les violences urbaines, le 29 février 1968, Johnson déclare : « Notre nation s'achemine vers une situation de deux sociétés, l'une noire et l'autre blanche, séparées et inégales ».

Martin Luther King, Jr. entame une campagne en faveur des pauvres (Poor People's Campaign). En 1967, il avait déclaré qu'il devait y avoir une « redistribution radicale du pouvoir économique et politique $»^{7}$. Dans le cadre de cette campagne, King se trouvait à Memphis (Tennessee) pour défendre les droits des éboueurs, tous noirs, salariés de la ville, lorsqu'il a été abattu sur le balcon de son motel par un assassin blanc, le 4 avril 1968.

13 La rage et le désespoir ressentis par beaucoup de Noirs à l'échelle nationale ont engendré des violences dans plus de 160 villes. Une nette radicalisation de la jeunesse noire des quartiers ghettoïsés a été remarquée lors de ces soulèvements, face aux conditions socio-économiques, face aux brutalités policières, mais aussi contre la guerre au Vietnam, car ce mouvement, en 1968, n'était plus l'apanage des étudiants et des intellectuels.

14 En 1968 le Black Panther Party devient une force d'attraction pour toute une jeunesse noire urbaine. Cette organisation, fondée à Oakland en 1966, existait dans plus de soixante villes deux ans plus tard et a continué à croître jusqu'en $1970^{8}$. Le procès politiquement marqué d'un des fondateurs du parti, Huey Newton, pour le meurtre 
présumé en 1967 d'un policier à Oakland, a été un point focal de l'actualité politique à l'été $1968^{9}$.

Les Panthers et le Southern Non-Violent Coordinating Committee (SNCC), en voie de radicalisation depuis 1965 sous la direction de Stokely Carmichael, annoncent une fusion début 1968. Ses bases politiques sont peu solides et elle ne dure pas longtemps, mais c'est un autre signe d'un processus de radicalisation ${ }^{10}$. À l'automne 1968, Hoover déclare que les Panthers sont une menace pour la sécurité intérieure des États-Unis. Au cours des années suivantes, des centaines de membres seront arrêtés. Pour la seule année 1969, 27 seront abattus par la police ${ }^{11}$.

Différents courants se réclamant du Black Power voient le jour, se distribuant sur une gamme allant du plus «nationaliste » au plus anti-impérialiste et marxisant. 1968 est également l'année d'une grève sauvage (wildcat strike) à l'usine principale de Dodge organisée par un groupe de révolutionnaires noirs, le Dodge Revolutionary Union Movement (DRUM) qui s'oppose au racisme non seulement dans les entreprises mais aussi dans les syndicats ${ }^{12}$.

17 Le sentiment de révolte se diffuse par des actes symboliques de défiance. Aux Jeux Olympiques de Mexico, le 16 octobre 1968, deux athlètes afro-américains, Tommie Smith et John Carlos, lèvent le poing en signe d'opposition au racisme aux Etats-Unis au moment de recevoir leurs médailles. L'année précédente, le champion de boxe Muhammad Ali avait déclaré son intention de ne pas participer à la guerre du Vietnam - car « aucun Vietnamien ne m'a jamais traité de sale nègre »-, acte pour lequel il a été privé de son titre de champion.

\section{Mouvements et mouvement : Chicanos, peuples indigènes, femmes, étudiants}

Le mouvement chicano (mexicain-américain), multiforme et polycentrique, était actif bien avant 1968, parmi les travailleurs agricoles en Californie, les petits agriculteurs du nord-ouest du Nouveau Mexique, ou la jeunesse urbaine des barrios du Sud-Ouest. En 1968 se multiplient les associations d'étudiants mexicains-américains sur les campus universitaires. Mais le grand événement de l'année a été la grève des lycéens de Los Angeles (walkouts ou blowouts) qui a commencé le 3 mars au lycée Abraham Lincoln du barrio d'East Los Angeles et a duré plusieurs semaines. La lutte des Farm Workers est marquée en février 1968 par une grève de la faim de César Chávez conçue comme un «appel au sacrifice» et à la non-violence. Plusieurs organisations du mouvement chicano participent en 1968 à la Poor People's Campaign lancée par King au moment de sa $\operatorname{mort}^{13}$.

Pour le mouvement des indigènes (Native Americans), 1968 fut l'année de la fondation, dans le Minnesota, de l'American Indian Movement (AIM). Ses actions spectaculaires et ses grands rassemblements commencent l'année suivante.

L'année 1968 peut être considéré comme un moment d'émergences pour le féminisme, où de nombreux groupes autonomes de femmes se forment et commencent à se fédérer à l'échelle nationale ${ }^{14}$. Notes from the First Year, la première revue théorique du féminisme américain, naît à New York. On relève un événement hautement symbolique des revendications du féminisme de la «seconde vague»: l'«invasion» et 
détournement du concours de beauté « Miss America » à Atlantic City (New Jersey), le 7 septembre. Parallèlement, le NAACP a animé un défilé « Miss Black America ».

Selon Katsiaficas, les mouvements contestataires des étudiants étaient devenus en 1968 «la règle plutôt que l'exception». Ce sont surtout des protagonistes blancs qui occupent l'avant-scène de l'actualité. Cependant, début février, à Orangeburg (Caroline du Sud), trois étudiants noirs de South Carolina State University sont tués et 33 blessés en manifestant devant un bowling qui ne les laissait pas entrer. L'incident passe largement inaperçu. En mars à Howard University, institution «historiquement noire " à Washington, D.C., des étudiants occupent le bâtiment de l'administration pendant quatre jours en exigeant le maintien de l'identité noire de l'établissement et un meilleur enseignement de l'histoire des Afro-Américains.

Cependant, le mouvement étudiant qui a défrayé la chronique était celui de Columbia, université prestigieuse de New York. À partir du 23 avril, quelques jours après l'assassinat de King, plus de 1000 étudiants occupent plusieurs bâtiments et le bureau du président. Ils contestent l'expansion annoncée du campus aux dépens des résidents afro-américains du secteur et la collaboration de l'université avec l'industrie militaire. Les étudiants sont délogés manu militari : 1250 blessés, 700 arrestations, beaucoup de violence des policiers contre de simples passants. L'événement a marqué une génération de militants. On connaît le rôle des étudiants de la majorité blanche, on commence à mieux connaître le rôle central d'un groupe d'étudiants noirs ${ }^{15}$.

Le SDS était encore en pleine expansion, passant de 30000 membres environ à l'automne 1967 à 80000 voire 100000 en novembre $1968^{16}$. Mais cette radicalisation et cette expansion s'accompagnèrent de graves divisions qui font rapidement éclater l'organisation en deux tendances professant chacune une forme de marxismeléninisme. Une scission groupusculaire par rapport à la deuxième tendance a donné naissance au Weather Underground dont les membres s'imaginaient en armée blanche d'alliés du mouvement de la libération noire (mais celui-ci n'en voulait pas). Lorsque, en 1970, le SDS disparait, le mouvement étudiant est privé d'une organisation nationale puissante.

24 Mais ce mouvement ne se limitait pas à SDS, organisation dont presque tous les membres étaient blancs. La grève du San Francisco State College, à partir de décembre 1968, sera le véritable lancement de tous les mouvements en faveur de programmes de Black Studies, Latino Studies et Native American Studies. Cette lutte s'est caractérisée par une alliance sous le signe du «tiers-monde » entre étudiants de diverses origines ${ }^{17}$.

\section{Politique électorale : le retour de bâton}

L'annonce de non candidature de Lyndon Johnson encourage le mouvement antiguerre. Le Sénateur Eugene McCarthy du Minnesota, figure peu connue, devient rapidement l'espoir d'une génération de jeunes en s'imposant dans les primaires. Robert Kennedy, frère du président assassiné, entrevoyant aussi sa chance, se présente dans les primaires en plaidant lui aussi pour le retrait des troupes. Il venait de gagner l'élection primaire de la Californie quand un assassin l'a abattu le 5 juin à Los Angeles.

26 La manifestation dans la rue en face de la Convention du Parti démocrate à Chicago, le 28 août 1968, a été un désastre médiatique pour ce parti et pour le mouvement. Les manifestants étaient peu nombreux, mais parmi les plus « enragés ». Le maire Richard 
Daley, « boss » autoritaire (et Démocrate pourtant), déploie 12000 policiers et fait venir 15000 autres agents territoriaux ou fédéraux. Sous les caméras de la télévision, la police se déchaîne contre les manifestants mais aussi contre journalistes et médecins venus en aide. Ces violences incontrôlées projettent une image de chaos dont ni les Démocrates ni la gauche émergente n'avaient besoin.

Réunis en Convention au même moment, les Démocrates se déchirent. Une résolution contre la guerre est écartée. Le vice-président Hubert Humphrey, ex-sénateur, investi sans passer par les élections primaires, sera affaibli par son incapacité à mobiliser les jeunes électeurs antiguerre.

Nixon, deux fois vice-président sous Eisenhower, gagne l'investiture du Parti républicain début août. Il saisit une opportunité en sollicitant les voix des Blancs sudistes restés opposés aux droits civiques. La marge de victoire de Nixon est extrêmement étroite en suffrages : $43,4 \%$ contre $42,7 \%$ pour Humphrey, même si le système du collège électoral donne au Républicain une victoire plus décisive : 301 "grands électeurs" contre 191. Mais si l'on ajoute au score de Nixon les 13,5\% du troisième candidat, le gouverneur George Wallace de l'Alabama, partisan tonitruant de la ségrégation raciale, ces $57,9 \%$ expriment plus nettement un tournant conservateur. La "majorité silencieuse», figure de la rhétorique nixonienne, est implicitement blanche et en colère contre les contestataires et les intellectuels.

\section{Rapport au présent, versants droite et gauche}

Lorsque de nombreux électeurs blancs du Sud abandonnent les Démocrates pour les Républicains, la logique de polarisation ethnoraciale est introduite au cœur de la vie politique partisane par un contre-mouvement de refus de l'égalité des droits ${ }^{18}$. Il serait exagéré de prétendre qu'il existe une ligne droite reliant Richard Nixon à Donald Trump, mais dès 1968 la polarisation racialisée transforme le Parti républicain en parti où le conservatisme implique la défense d'une hégémonie blanche.

Si, à partir de 1968, la Nouvelle Gauche s'affaiblit et se disloque (thèse de Gitlin), la lecture d'Elbaum permet de voir que la défaite du mouvement n'a jamais été totale. Des forces anticapitalistes et antiracistes non négligeables ont, pour un temps encore, continué à se construire et à mener des campagnes. Les "nouveaux communistes " dans les années 1970 ont construit des organisations de taille réduite dont le discours idéologique était parfois très sectaire, mais ces groupements étaient néanmoins d'importants viviers de militants sans lesquels ni le mouvement contre l'intervention de l'administration Reagan en Amérique centrale dans les années 1980, ni la campagne social-démocrate de la Rainbow Coalition de Jesse Jackson dans les élections primaires démocrates $(1984,1988)$, n'auraient connu un tel impact ${ }^{19}$. L'argumentation d'Elbaum pourrait s'appliquer aussi à d'autres courants de gauche, davantage sociauxdémocrates, issu des mouvements de 1968. Peut-on voir indirectement dans cette présence la genèse du mouvement Occupy Wall Street (2011), aux succès du sénateur Bernie Sanders dans les primaires démocrates (2016), au mouvement \#BlackLivesMatter (2013) ? De futures recherches pourraient révéler, à gauche aussi, des continuités insoupçonnées depuis cinquante ans. 


\section{NOTES}

1. Max Elbaum, Revolution in the Air: Sixties Radicals turn to Lenin, Mao and Che, Verso, 2002, p. 40.

2. Ibid., p. 37.

3. Geroge Katsiaficas, The Imagination of the New Left: A Global Analysis of 1968, South End Press, 1987.

4. Joshua B. Freeman, American Empire, 1945-2000: The Rise of a Global Power, the Democratic Revolution at Home, Penguin Books, 2012, p. 241-246.

5. Todd Gitlin, The Sixties: Years of Hope, Days of Rage, Bantam, 1987, chapitre 12.

6. Ibid., p. 293.

7. https://www.poorpeoplescampaign.org/index.php/poor-peoples-campaign-1968/

8. Joshua Bloom et Waldo E. Martin, Jr., Black Against Empire: The History and Politics of the Black Panther Party, University of California Press, 2013, p. 2.

9. Todd Gitlin, op. cit., chapitre 12.

10. Ibid., p. 111-114.

11. Max Elbaum, op. cit., p. 66

12. Ibid., p. 80-82.

13. Juan Gómez Quiñones, Chicano Politics: Reality and Promise, 1940-1990, University of New Mexico Press, 1990, p. 114.

14. George Katsiaficas, op. cit., p. 76-77

15. Paul Cronin (organisateur), A Time to Stir: Columbia '68, Columbia University Press, 2018

16. Max Elbaum, op. cit., p. 69.

17. Rolland-Diamond Caroline, "Sociohistoire des Black Studies Departments", IdeAs n 2, 2012. URL: https://journals.openedition.org/ideas/266

18. Doug McAdam et Karina Kloos, Deeply Divided: Racial Potlics and Social Movements in Postwar America, Oxford University Press, 2014.

19. Max Elbaum, op. cit., chapitres 12-13. 